\title{
European Women Shareholders Demand Gender Equality: der djb unterwegs in Europa
}

Erfolg: Das djb-Projekt „European Women Shareholders Demand Gender Equality “ hat im April 2014 von der Europäischen Kommission neben 16 weiteren europäischen Projekten den Zuschlag für eine Förderung nach dem PROGRESS-Programm erhalten. Es wird kofinanziert vom Bundesministerium für Familie, Senioren, Frauen und Jugend (BMFSFJ), dem Ministerium für Justiz und Gleichstellung des Landes Sachsen-Anhalt und der Finnischen Handelskammer. Am 16. Mai 2014 war offizieller Projektbeginn.

Ein gerechter Anteil der Frauen an Führungspositionen der Wirtschaft steht sowohl in Deutschland als auch in anderen Mitgliedstaaten der Europäischen Union seit längerer Zeit auf der Tagesordnung. Der djb ist in die Offensive gegangen und will eine Auseinandersetzung über die Unternehmenskultur und Chancengleichheit von Frauen und Männern bei Führungspositionen der Wirtschaft auch in europäischen Unternehmen anstoßen. Die Idee dafür gab das vom BMFSFJ von 2009 bis 2014 finanzierte Projekt Aktionärinnen fordern Gleichberechtigung (s. S. 3ff. in diesem Heft): Im Rahmen dieses Projektes hat der djb zwischen 2009 und 2014 mehr als 300 Hauptversammlungen besucht und kritische Fragen zur Karriereentwicklung und Vertretung der Frauen in Führungspositionen gestellt. Eine ähnliche Aktion wird von Juristinnen in Spanien durchgeführt. Vor diesem Hintergrund sollen in einem nächsten Schritt die spezifischen Erfahrungen mit Kollegen/innen in anderen EU-Mitgliedstaaten geteilt werden. Im ersten Halbjahr 2015 werden europaweit Hauptversammlungen großer Unternehmen, darunter alle EURO STOXX 50-Index-Unternehmen - in den folgenden Mitgliedstaaten besucht: Benelux, Bulgarien, Deutschland, Spanien, Frankreich, Italien, Großbritannien und Irland. Den Board-Mitgliedern sollen detaillierte und auf das konkrete Unternehmen bezogene Fragen nach Frauen in
Führungspositionen und nach deren Karrieremöglichkeiten gestellt. Zur Vorbereitung auf die Besuche dienen Workshops und Toolkits mit Fragenkatalog, Erfahrungsberichten, FAQs usw. In Finnland werden keine Hauptversammlungen besucht, sondern die finnische Handelskammer stellt ihre eigenen Erfahrungen im Sinne von best practice zur Verfügung (direkter Kontakt zu den Unternehmen die Mangel an Gleichstellung auf die Führungsebenen aufweisen).

Das Projekt unterstützt die Bemühungen der Europäischen Kommission um eine angemessene Repräsentation von Frauen in Leitungspositionen der Privatwirtschaft. Die Kommission hatte Ende 2012 eine Richtlinie vorgeschlagen, die u.a. den rund 5.000 börsennotierten Firmen in der EU vorschreibt, bis 2020 Aufsichtsratsposten zu 40 Prozent mit Frauen zu besetzen.

Seit Mitte Mai 2014 ist auch das für die Verwaltung und Koordination der europaweiten Aktivitäten zuständige Projektbüro in Berlin eingerichtet. Es hat mit Miruna Bucurescu (Projektmitarbeiterin) und Linda Walczak (Sachbearbeiterin) zwei Mitarbeiterinnen, die ihre eigene rumänische und polnische EU-Perspektive einbringen können. Zwei EU-Mitgliedstaaten, die im Projekt als Partner bislang nicht vertreten waren. Das Projektbüro ist per E-Mail eu-womnenshareholders@djb.de und telefonisch (Tel. +49 30 46798600) erreichbar.

$\mathrm{Zu}$ den Projektpartnern gehören neben dem djb: die vier Juristinnenorganisationen EWLA (European Women Lawyers Association), EWLA Bulgarien, AFFJ (Association française des femmes juristes), der Ungarische Anwältinnenverband sowie die Finnische Handelskammer und die Universität von Westengland. Das Ministerium für Justiz und Gleichstellung des Landes Sachsen-Anhalt ist Gastgeber der Abschlusskonferenz in Brüssel Anfang 2016 und die Universität Magdeburg Stendal ist für Medienarbeit zuständig.

\section{Bewerbungen für den Marie Elisabeth Lüders-Preis 2015}

Der Deutsche Juristinnenbund e.V. (djb) vergibt in Anerkennung hervorragender rechts- oder wirtschaftswissenschaftlicher Arbeiten alle zwei Jahre den Marie Elisabeth Lüders-Preis. Ausgezeichnet werden Rechts- und Wirtschaftswissenschaftlerinnen. Preiswürdig sind rechts- oder wirtschaftswissenschaftliche Dissertationen und Habilitationsschriften zum Bereich Recht und Geschlecht sowie Arbeiten, die im Zusammenhang mit dem Thema Gleichstellung von Frau und Mann deutliche rechts- oder wirtschaftswissenschaftliche Bezüge aufweisen. Die Mitgliedschaft im djb ist keine Voraussetzung. Der Preis besteht aus einem Druckkostenzuschuss in Höhe von 2.000,- Euro und wird alle zwei Jahre durch die Präsidentin im Rahmen der Mitgliederversammlung verliehen,

Bewerbungen für den Marie Elisabeth Lüders-Preis 2015 sind bis zum 31. August 2014 bei der Präsidentin des djb, Bundesgeschäftsstelle des djb, Anklamer Straße 38, 10115 Berlin einzureichen. Weitere Informationen stehen in der Preisordnung (http://www.djb.de/Wissenschaftspreis/Preisordnung/) 


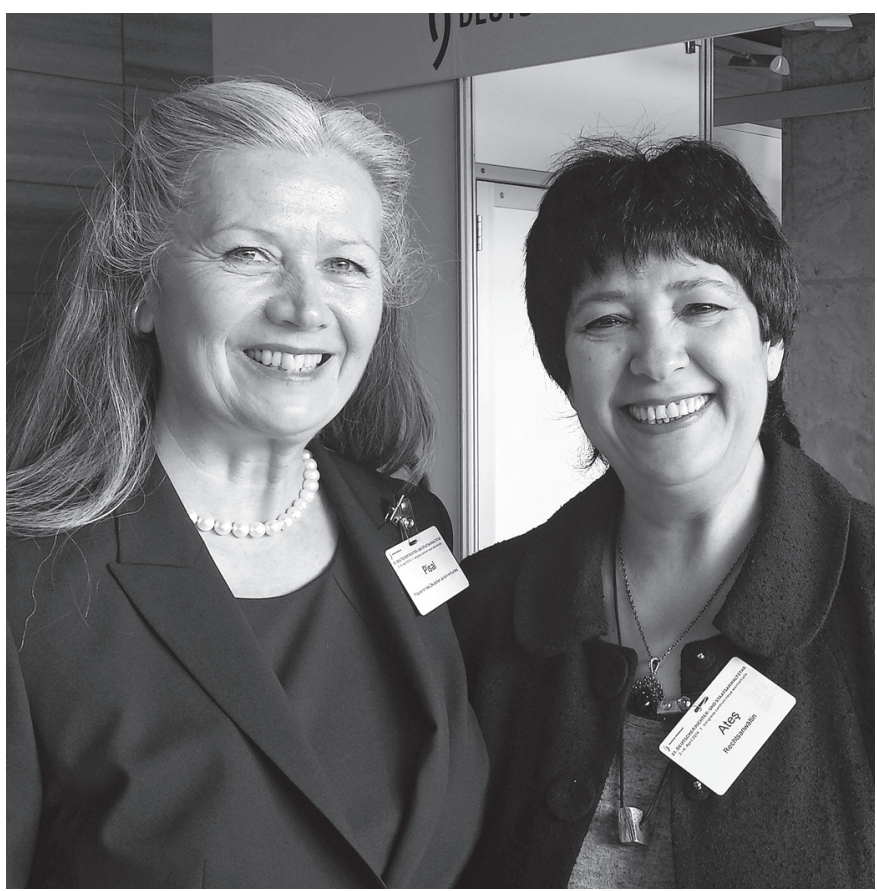

४ djb-Präsidentin Ramona Pisal und Rechtsanwältin Seyran Ateș, Berlin, beim 21. Deutschen Richter- und Staatsanwaltstag „Grenzen des Rechts Recht ohne Grenzen" vom 2. bis 4. April 2014 in Weimar. Seyran Ateș war Podiumsteilnehmerin des Streitpunktes II „Scharia - Parallele Gesellschaft, eigene Justiz?“ (@ AG/djb, Berlin).

Insgesamt waren knapp 1000 Besucher in der ersten Aprilwoche der Einladung des DRB gefolgt und in die Kulturmetropole Weimar gereist, um an den Veranstaltungen des RiStA-Tages teilzunehmen. In vier Streitpunkten, 21 Workshops, dem Forum Gerechtigkeit und bei der Schlussveranstaltung sind drei Tage lang aktuelle rechtspolitische Vorhaben und praxisrelevante Probleme beleuchtet und kontrovers diskutiert worden. So die durch den Fall Hoeneß wieder in den Fokus der Öffentlichkeit gerückte strafbefreiende Selbstanzeige, die Thematik der islamischen Paralleljustiz in Deutschland oder die Probleme, die an der Schnittstelle zwischen Medizin und Recht entstehen.

\section{Geburtstage}

(Januar bis März 2014)

\section{Jahre}

- Hildegard Bodendieck-Engels Vorsitzende Richterin am LG a.D. Strande

- Dr. Ulrike von Dellingshausen Vorsitzende Richterin am LG Münster

narie-Luise Gastroph Richterin am OLG a.D. München

\section{- Regina Hermisson} Richterin am OLG Freiburg

Dr. Bettina Lange-Klein Ministerialdirigentin Bonn

\section{- Doretta Loschelder} Botschafterin i.R. Berlin
- Katrin Markus Rechtsanwältin

Düsseldorf

\section{Jahre}

- Gisela Finzel

Richterin am AG i.R.

Bielefeld

- Magretlies Neumann-Niederschlag Rechtsanwältin und Notarin a.D. Oldenburg

- Dr. Irmgard Ohl-Stauffer Karlsruhe

\section{8o Jahre}

- Prof. Dr. Dr. h. c. Jutta Limbach Präsidentin des BVerfG i.R. Berlin

\section{Verstorben}

(Januar bis März 2014)

- Ute Hanisch Rechtsanwältin Neubrandenburg im 67. Lebensjahr 